\title{
Crystallization Kinetics of Cocoa Fat Systems \\ Experiments and Modeling
}

\author{
Journal Article \\ Author(s): \\ Padar, S.; Jeelani, S. A.K.; Windhab, Erich J. \\ Publication date: \\ 2008-12 \\ Permanent link: \\ https://doi.org/10.3929/ethz-b-000009583
}

Rights / license:

In Copyright - Non-Commercial Use Permitted

Originally published in:

Journal of the American Oil Chemists' Society 85(12), https://doi.org/10.1007/s11746-008-1312-0 


\title{
Crystallization Kinetics of Cocoa Fat Systems: Experiments and Modeling
}

\author{
S. Padar $\cdot$ S. A. K. Jeelani $\cdot$ E. J. Windhab
}

Received: 5 June 2008/Revised: 18 September 2008/ Accepted: 2 October 2008/Published online: 7 November 2008 (C) AOCS 2008

\begin{abstract}
Isothermal crystallization kinetics of unseeded and seeded cocoa butter and milk chocolate is experimentally investigated under quiescent conditions at different temperatures in terms of the temporal increase in the solid fat content. The theoretical equations of Avrami based on one-, two- and three-dimensional crystal growth are tested with the experimental data. The equation for onedimensional crystal growth represents well the kinetics of unseeded cocoa butter crystallization of form $\alpha$ and $\beta^{\prime}$. This is also true for cocoa butter crystal seeded milk chocolate. The sterical hindrance due to high solids content in chocolate restricts crystallization to lineal growth. In contrast, the equation for two-dimensional crystal growth fits best the seeded cocoa butter crystallization kinetics. However, a transition from three- to one-dimensional growth kinetics seems to occur. Published data on crystallization of a single component involving spherulite crystals are represented well by Avrami's three-dimensional theoretical equation. The theoretical equations enable the determination of the fundamental crystallization parameters such as the probability of nucleation and the number density of nuclei based on the measured crystal growth rate. This is not possible with Avrami's approximate equation although it fits the experimental data well. The crystallization can be reasonably well defined for single component systems. However, there is no model which fits the multicomponent crystallization processes as observed in fat systems.
\end{abstract}

S. Padar $(\bowtie) \cdot$ S. A. K. Jeelani · E. J. Windhab Laboratory of Food Process Engineering, Institute of Food Science and Nutrition,

Swiss Federal Institute of Technology (ETH Zurich),

Schmelzbergstrasse 9, 8092 Zürich, Switzerland

e-mail: stefan.padar@ilw.agrl.ethz.ch
Keywords Avrami - Chocolate - Cocoa butter . Crystallization - Nucleation - Seeding · Solid fat content Triacylglycerol

\section{List of symbols}

$k$ rate constant in Avrami approximate equation $\left(\mathrm{s}^{-m}\right)$ $m$ exponent in Avrami approximate equation

$N \quad$ number of germ nuclei per unit volume at any instant of time $\left(\mathrm{m}^{-3}\right)$

$N_{0} \quad$ initial number of germ nuclei per unit volume $\left(\mathrm{m}^{-3}\right)$

$p \quad$ probability of growth of germ nuclei per unit time $\left(\mathrm{s}^{-1}\right)$

$l \quad$ length or radius of crystal grain (m)

$i$ average rate of growth of length or radius of crystal grain $\left(\mathrm{m} \mathrm{s}^{-1}\right)$

$S \quad$ mass of crystalline phase per unit mass of lipid phase

$S_{m} \quad$ maximum mass of crystalline phase per unit mass of lipid phase

$S_{m 0}$ fraction of solid fat at the beginning of bulk crystallization

$t$ time (s)

$T$ temperature (K)

$v \quad$ volume of crystal grain $\left(\mathrm{m}^{3}\right)$

$V \quad$ volume of crystalline phase per unit volume of suspension

$V_{m} \quad$ maximum volume of crystalline phase per unit volume of suspension

$V_{e} \quad$ volume of crystal grains per unit volume of suspension formed without growth impediment

$z \quad$ dimensionless re-scaled time

\section{Greek letters}

$\dot{\gamma}$ shear rate $\left(\mathrm{s}^{-1}\right)$

$\sigma_{1}$ cross-sectional area of rod-like crystal $\left(\mathrm{m}^{2}\right)$

$\sigma_{2}$ thickness of plate-like crystal (m) 
$\sigma_{3} \quad$ shape factor for sphere-like crystal

$\eta \quad$ dynamic viscosity ( $\mathrm{Pa} \mathrm{s}$ )

$\tau \quad$ dimensionless characteristic time $p t$

\section{Introduction}

Chocolate and many confectionery products contain cocoa butter, which is a mixture of triacylglycerols (TAG) of stearic, palmitic, oleic and other fatty acids. The physical and textural properties of this food ingredient depend on the microstructure formed during the crystallization of liquid fat below the respective melting temperature and are affected by the processing and storage conditions. Fats, in general, feature a complex polymorphism, which is strongly influenced by its TAG composition and heat, mass, and momentum transfer during crystallization. The three main fat crystal polymorphs are, in order of increasing stability, the $\alpha$ (hexagonal subcell), several $\beta^{\prime}$ (orthorhombic), and several $\beta$ (triclinic) forms [1].

The fat phase in chocolate mainly consists of cocoa butter. At room temperature it is in a crystalline solid state. Polymorphism of cocoa butter is an important material characteristic in chocolate manufacturing as it is directly linked to product quality and processing performance. From the six crystal forms confirmed in cocoa butter [2,3] only form $\beta_{\mathrm{V}}$-the second most stable crystal form-is desired in the final product. For initiating this crystal form, a precrystallization process has to be applied before the chocolate is molded and solidified. Without introducing crystals of $\beta$-type prior to cooling, the fat phase in chocolate would solidify in the less stable crystal form $\alpha$ under industrial processing conditions. The subsequent transformation into the higher melting $\beta^{\prime}$ polymorph $\left(\beta_{\text {IV }}\right)$ followed by the two $\beta$ crystal forms ( $\beta_{\mathrm{V}}$ and $\beta_{\mathrm{VI}}$ ) would lead to a complete loss of the organoleptic properties of commercial chocolate. Hence, quiescent experiments for characterizing the crystallization in form $\beta_{\mathrm{V}}$ need to be carried out with precrystallized samples.

Himawan et al. [4] recently reviewed the various thermodynamic and kinetic aspects of fat crystallization and emphasized that the complexity of crystallization of fats is associated with not only the polymorphism but also the several components of the fat system. Since cocoa butter is a mixture of TAGs, a melting range exists rather than a melting point [5]. Consequently, a temperature and time dependence of the mass fraction of solid fat is observed and is quantified by the solid fat content (SFC).

The experimental investigation of the kinetics of isothermal crystallization is usually carried out by measuring the time dependency of the SFC, which is often a sigmoidal curve. Deterministic, numerical and stochastic models [68] have been developed for the interpretation of the experimental results. Avrami $[9,10]$ was the first to present a deterministic model for single component isothermal crystallization, which allowed for instantaneous nucleation and growth, and impingement of crystal grains. He derived theoretical equations for the increase with time in the volume fraction of crystallized phase for linear onedimensional (1-D), plate-like two-dimensional (2-D) and sphere-like three-dimensional (3-D) growth of crystal grains. By truncating the power series of an exponential function of time and replacing it by a power law, he obtained an approximated equation (referred in the present paper as the Avrami approximate equation).

Avrami $[9,10]$ developed the model for the kinetics of crystallization of low molecular weight materials such as metals. It was subsequently extended to the crystallization of different high molecular weight polymers by Mandelkern et al. [11] and to palm oil (a mixture of triacylglycerides) by Kawamura [12]. Although the experimental sigmoidal crystallization curve was usually well fitted by the Avrami approximate equation, the two parameters involved are empirical and do not directly explain crystallization physically. In spite of this, Avrami's approximate equation was widely used. Several explanations were deduced how nucleation kinetics affected the Avrami exponent. Sharples [13] used a first order nucleation kinetics to introduce sporadic nucleation. Impingement, however, was neglected. Marangoni [14] applied an analogy theory using the chemical diffusion equation based on Fick's first law. The increase in solid content was calculated from the change in surface area. These approaches made assumptions based on analogies instead of physical evidence. The assumption of a linear temporal increase in number of nuclei during sporadic nucleation and ignoring the effect of impingement can only be applied to the early stage of crystallization when the crystal concentration is low. Consequently, such assumptions are not realistic at high crystal concentrations. Several authors [8, 13-15] have tabulated the values of the exponent in Avrami's approximate equation corresponding to the nucleation kinetics and shape of the crystals. Sharples [13] gave causes for the limitation of this classification. Spatially not randomly distributed nucleation, time dependence of the nucleation or the growth-process or changes in density of the growing bodies can result in a continually changing value of the Avrami exponent. Moreover, the simultaneous development of different crystal forms and growth of similar crystals from different types of nuclei (sporadic and instantaneous) are mentioned by Christian [15]. Marangoni [14] identified remarkable changes of the Avrami exponent by the degree of supercooling for milk fat crystallization. All these aspects were considered to contribute to the fractional nature of the exponent. Hence, the same values of the Avrami exponents 
may be obtained as a result of different mechanisms [16]. Deeper insight into the fundamental relationships during crystallization is necessary for improving the existing models. Kinetic aspects, for example, the diffusion rate of the molecules within the liquid, the orientation or the attachment are as important as thermodynamic factors [4].

Some authors [17, 18] reparameterized Avrami's approximate equation. The so called Erofeev model was used by $\mathrm{Ng}$ [17] to describe the thermal decomposition in the solid state. Marangoni [19] considered the reparameterization of Avrami's approximate equation by these authors was arbitrary and did not have any theoretical justification.

Another explanation for fractional values of the Avrami exponent was stated by Liu and Sawant [20]. When applying Avrami's approximate equation to a supramolecular material which is building up a fractal fibrous network during gelation, they could show that the exponent is related to the fractal dimension of branching events. Marangoni and Ollivon [21] presented the fractal nature of TAG spherulites due to nucleation kinetics in a non crystallizing diluent.

Foubert et al. [7, 8] proposed a deterministic mathematical model by analogy with reversible reaction kinetics, which generally fits well with experimental sigmoidal data but the parameters do not have as much physical meaning as those of the Avrami model. Other deterministic models derived for the rates of flocculation and aggregation of colloidal particles in liquids, and rates of bacterial growth were used by Berg and Brimberg [22], and Kloek et al. [23] respectively to fit the experimental crystallization results. These authors claimed an analogy with the crystallization mechanisms.

The numerical model, although theoretically similar to the deterministic model, uses complicated equations for nucleation and growth rates of crystal grains. Consequently, the resulting equations, which cannot be solved analytically, must be numerically integrated [6]. In contrast, stochastic models consider nucleation and growth as statistical or probabilistic events. A two- or three-dimensional space is defined in which the temporal evolution of the solid/liquid interface, and hence the solid content, can be obtained.

The present paper investigates experimentally the kinetics of isothermal crystallization of precrystallized cocoa butter and milk chocolate. This involved two precrystallization techniques-seeding with cocoa butter crystal suspension (CBCS) and conventional chocolate tempering. In addition, unseeded cocoa butter crystallization kinetics is investigated. We demonstrate that only one of the three theoretical equations of Avrami fits well the experimental single component crystallization kinetics data. This provides the fact that the crystallization is either in 1D, 2-D or 3-D. In contrast, although Avrami's approximate equation also fits the data, it does not enable us to know the exact type of crystal growth. We confirm the method of theoretical analysis using the experimental data published by Manchado et al. [24] on the kinetics of crystallization of polypropylene melt involving growth of spherulite-shaped crystal grains. This is corroborated by the microscopic information of the shape and rate of crystal growth. In contrast, we find fractionation during crystallization of multicomponent cocoa fat systems and the observed bimodal kinetics is described by double-Avrami fits.

\section{Modeling of Isothermal Crystallization}

As discussed above, there are deterministic, numerical and stochastic models for the kinetics of crystallization. The present work focuses on deterministic models such as that of Avrami, which is described below for the sake of better understanding.

\section{Avrami's Theoretical Model}

Avrami's [9, 10] model for the kinetics of isothermal crystallization allowed for nucleation, crystal growth and impingement. Nucleation was considered to be either sporadic (in which the number $N$ of nuclei per unit volume increases linearly with time) or instantaneous (in which $N$ is created initially which is equivalent to a seeded crystallization). After formation, the radii of the nuclei were assumed to grow at a constant rate to determine the volume of crystals, which did not involve impingement. Avrami subsequently allowed for the impingement of the crystals, a summary of the equations is given below.

The number density $N$, decreases with time $t$ as some nuclei grow due to free energy fluctuations with probability of occurrence $p$ per unit time and some impinge with other nuclei. If $v(\tau, z)$ is the volume of a crystal grain at a characteristic time $\tau=p t$, which grows from a nucleus at time $z$, then the total extended volume $V_{e}$ (representing the volume of the grains if their growth is unimpeded) of crystals per unit volume of suspension is given by

$V_{e}=\int_{0}^{\tau} v(\tau, z) N(z) \mathrm{d} z$

The number density $N(z)$ of grains was assumed to decrease exponentially with time $z$ as:

$N(z)=N_{0} \mathrm{e}^{-z}$

so that Eq. 1 becomes

$V_{e}=N_{0} \int_{0}^{\tau} v(\tau, z) \mathrm{e}^{-z} \mathrm{~d} z$ 
In order to allow for the impingement of crystals, Avrami assumed that the volume of crystals increases linearly with the volume fraction of remaining liquid and obtained the following equation for the volume $V$ of the crystalline phase per unit volume of suspension:

$V=1-\mathrm{e}^{-V_{e}}$

This enables the determination of the kinetics of crystallization in which $V_{e}$ can be obtained using Eq. 3. The latter, in turn, is determined by the time dependent increase in the volume $v(\tau, z)$ of crystal grains as discussed below.

The crystal grains can grow in one or two or three dimensions, respectively termed as lineal (rod-like), platelike or pseudospherical (or polyhedral) shapes. The length $l$ of the crystal grain growing at a rate $i$ at time $\tau$, which grows from a nucleus at time $z$, is given by:

$l(\tau, z)=\int_{z}^{\tau} \frac{i}{p} \mathrm{~d} u$

The factors influencing the growth of the nuclei and crystal grains were considered to be similar so that $p$ and $i$ were assumed to be proportional over the whole range of temperature and concentration. Thus, assuming $i / p$ to be constant for a substance in the isokinetic range, Avrami obtained:

$l(\tau, z)=\frac{i}{p}(\tau-z)$

He also assumed the rates of growth to be the same in all directions for crystal grains growing in two and three dimensions. Thus the volumes of crystal grains growing in one or two or three dimensions, are respectively given by

$v(\tau, z)=\sigma_{1} l=\frac{\sigma_{1} \dot{l}}{p}(\tau-z)$

$v(\tau, z)=\sigma_{2} l^{2}=\frac{\sigma_{2} \dot{l}^{2}}{p^{2}}(\tau-z)^{2}$

$v(\tau, z)=\sigma_{3} l^{3}=\frac{\sigma_{3} i^{3}}{p^{3}}(\tau-z)^{3}$

$\sigma_{1}$ is the cross-sectional area of the rod-like crystal grain growing along its length while $\sigma_{2}$ is the thickness of the plate-like crystal grain growing in two dimensions. For a crystal grain growing in three dimensions, $\sigma_{3}$ is the shape factor which is equal to $4 \pi / 3$ for a sphere.

Thus substitution of Eqs. 7, 8 and 9 in Eq. 3 and integration resulted in the total extended volume $V_{e}$ of crystal grains growing in one or two or three dimensions respectively as

$V_{e}=\frac{\sigma_{1} i N_{0}}{p}\left\{\mathrm{e}^{-\tau}-1+\tau\right\}$

$$
\begin{aligned}
& V_{e}=\frac{2 \sigma_{2} i^{2} N_{0}}{p^{2}}\left\{-\mathrm{e}^{-\tau}+1-\tau+\frac{\tau^{2}}{2}\right\} \\
& V_{e}=\frac{6 \sigma_{3} l^{3} N_{0}}{p^{3}}\left\{\mathrm{e}^{-\tau}-1+\tau-\frac{\tau^{2}}{2}+\frac{\tau^{3}}{6}\right\}
\end{aligned}
$$

which are valid until the time $\tau=\bar{\tau}$ of exhaustion of germ nuclei. Substituting the above equations in Eq. 4 gives the corresponding theoretical equations of Avrami for the volume fraction of crystalline solid phase for one or two- or threedimensional growth of crystals respectively. Equation 4 was derived assuming that the maximum volume fraction of crystallizable solid is unity for crystallization from melts. However, in general, the maximum volume fraction of the crystallized fat $V_{m}$ is less than unity, which depends on the degree of super-cooling or the presence of polymorph crystals or even impurities at the particular constant temperature. Consequently, Eq. 4 can be rewritten as

$V=V_{m}\left(1-\mathrm{e}^{-V_{e}}\right)$

Substituting Eqs. 10, 11 and 12 in Eq. 13 gives the volume fraction of crystalline solid for one- or two- or threedimensional growth of crystals respectively as

$$
\begin{aligned}
& V=V_{m}\left[1-\mathrm{e}^{-\left(\sigma_{1} i N_{0} / p\right)\left\{\mathrm{e}^{-\tau}-1+\tau\right\}}\right] \\
& V=V_{m}\left[1-\mathrm{e}^{-\left(2 \sigma_{2} i^{2} N_{0} / p^{2}\right)\left\{-\mathrm{e}^{-\tau}+1-\tau+\left(\tau^{2} / 2\right)\right\}}\right] \\
& V=V_{m}\left[1-\mathrm{e}^{-\left(6 \sigma_{3} l^{3} N_{0} / p^{3}\right)\left\{\mathrm{e}^{-\tau}-1+\tau-\left(\tau^{2} / 2\right)+\left(\tau^{3} / 6\right)\right\}}\right]
\end{aligned}
$$

in which $\tau=p t$. The above equations for one- or two- or three-dimensional growth of crystals can be also used in terms of the mass fraction of the crystallized solid $S$ by replacing $V$ and $V_{m}$ by $S$ and $S_{m}$ respectively, where $S_{m}$ is the maximum value of the mass fraction of the crystalline solid phase.

\section{Avrami's Approximate Equation}

Avrami obtained an approximate equation by considering two limiting cases when the initial number density of nuclei is large so that nuclei exist until the end of crystallization. When $\tau=p t$ is small, he truncated the terms higher than fourth order in the power series of the exponential term $\mathrm{e}^{-\tau}$, the first four terms of which cancelled with the four terms in the bracket of Eq. 16 for the threedimensional growth of crystals to obtain:

$V=V_{m}\left[1-\mathrm{e}^{-\left(6 \sigma_{3} l^{3} N_{0} / p^{3}\right)\left(\tau^{4} / 24\right)}\right]$

Very small values of $\tau=p t$ could be due to either small values of $p$ which he attributed to sporadic nucleation or small values of time $t$. 
When $\tau=p t$ is very large, due to very large values of $p$ which he attributed to instantaneous nucleation, he neglected the exponential term $\mathrm{e}^{-\tau}$ and the terms up to the order of $\tau^{2}$ in Eq. 16 to obtain:

$V=V_{m}\left[1-\mathrm{e}^{-\left(6 \sigma_{3} l^{3} N_{0} / p^{3}\right)\left(\tau^{3} / 6\right)}\right]$

As mentioned by Foubert et al. [8], Avrami did not consider the possibility of intermediate values of $\tau$. The relationship between $V$ and $\tau=p t$ for intermediate values of $\tau$ (due to intermediate values of $p$ and $t$ ) was assumed to lie between those described by Eqs. 17 and 18. Thus, in general, the following approximate equation for the whole range of $\tau$ was presented:

$V=V_{m}\left(1-\mathrm{e}^{-k t^{m}}\right)$

which is generally known as the Avrami equation. However, we would rather refer to this as the Avrami approximate equation in order to distinguish it from the Avrami theoretical Eqs. 14, 15 and 16 for one-, two- and three-dimensional growth of crystals respectively.

According to Avrami, the exponent $m$ should be an integer, the value of which depends on the type (sporadic or instantaneous) of nucleation and morphology of crystalline particles. The rate constant $k$ depends on the rates of nucleation and growth of crystal grains. However, fitting of experimental data to Eq. 19 often resulted in non-integer values of $m$, which have been explained by several authors $[13,15,25,26]$.

However, it is not clear why several authors have been using Avrami's approximate Eq. 19 for fitting and interpreting the experimental data on the kinetics of crystallization even though it does not provide the exact values of the nucleation rate parameters $p$ and $N_{0}$, and crystal growth rate $i$. In fact, the use of the theoretical Eqs. 14 or 15 or 16 of Avrami for fitting the experimental data on the variation in crystal content with time can give not only the information on whether the crystal growth is one- or two- or three-dimensional but also the exact values of the nucleation and growth parameters.

\section{Experimental}

\section{Material}

Cocoa butter was obtained from ADM Cacao B. V. (Netherlands). Its TAG composition (weight \%) evaluated by gas chromatography is as follows: $44.3 \%$ POS, $32.8 \%$ SOS, $17.7 \%$ POP, 3.2\% SOO, 2.0\% POO (P: palmitic, O: oleic and $\mathrm{S}$ : stearic fatty acid). Small quantities of other TAGs were not determined.

We used a commercial milk chocolate (Chocolats Halba AG, Switzerland) with $41.4 \%$ sugar, $23.2 \%$ whole milk powder, $17.7 \%$ cocoa mass, $15.9 \%$ cocoa butter, $1.3 \%$ skimmed milk powder and $0.5 \%$ soya lecithin.

\section{Precrystallization}

Two main processes exist for creating the $\beta_{\mathrm{V}}$ crystal nuclei in chocolate-generating the nuclei in the bulk by conventional mass tempering, and seeding with crystals in the crystal form to be favored, as described by Zeng [27]. For conventional precrystallization we used a chocolate temperer AMK50 (Aasted-Mikroverk ApS, Denmark). Concerning the generally applied quality specification for precrystallized chocolate [28], we adjusted the processing conditions to obtain a well tempered chocolate. All experiments with chocolate were performed at $28^{\circ} \mathrm{C}$ which was identical to the outflow temperature of the chocolate temperer.

In the experiments with seeded chocolate we compared two different devices. The single-stage SeedMaster CrystMix (Bühler AG, Switzerland) produces the CBCS batchwise while the two-stage SeedMaster Compact (Bühler AG, Switzerland) works continuously. In both processes the CBCS is manufactured in a scraped heat exchanger (first stage) at $15{ }^{\circ} \mathrm{C}$ wall temperature. In the single-stage system the subsequent conditioning step happens in a temperature controlled vessel for $2 \mathrm{~h}$ at $31.5^{\circ} \mathrm{C}$ applying low shear. In the continuous two-stage process conditioning is performed within the second stage of the scraped heat exchanger at high shear.

The cocoa butter suspension consists of finely dispersed $\beta_{\mathrm{V}}$-crystals with a SFC of $12 \%$ for the single-stage crystallizer. Based on the fat concentration in the sample we added $2.4 \%$ of CBCS for precrystallization (2.4\% for pure cocoa butter and $0.8 \%$ for chocolate) and used an anchor stirrer at $180 \mathrm{rpm}$ for $60 \mathrm{~s}$ to get a homogeneous distribution. An SFC of $17 \%$ was reached in the two-stage process. According to the criteria of well tempered chocolate we determined a seeding quantity of $0.4 \%$.

\section{Measuring Procedure}

We measured the SFC with a low resolution impulsional Bruker Minispec NMS 120 spectrometer operating at $20 \mathrm{MHz}$. Slight adaptions were necessary between the two measurement methods used-the direct and the indirect method, respectively [29, 30]. The filling height in the sampling tube of $10 \mathrm{~mm}$ diameter was approximately $40 \mathrm{~mm}$ for the direct and $10 \mathrm{~mm}$ for the indirect method. For each experiment we performed 6 or 8 replicates. The samples and the probe head were temperature controlled using external water baths. We investigated isothermal crystallization at 20 and $25^{\circ} \mathrm{C}$ for unseeded cocoa butter, 
at 28,30 and $32{ }^{\circ} \mathrm{C}$ for seeded cocoa butter and at $28{ }^{\circ} \mathrm{C}$ for precrystallized chocolate.

\section{Direct Method}

We applied the direct measuring method as described by van Putte and van den Enden [31]. The instrument calculates the SFC based on the calibration performed prior to the experiments. The calibration standards with defined solids contents (plastic material Celloron immersed in paraffin oil) have a relaxation behavior similar to fat systems. We determined a correction factor ( $f$-value) of 1.52 for approximating the initial signal intensity. Each sample was measured in 3 repetitions with a recycle delay of $6 \mathrm{~s}$. This is in accordance with the findings of van Putte and van den Enden [31], who proposed a prolongation of the recycle delay up to $8 \mathrm{~s}$ for higher crystal polymorphs. As summarized by Timms [32], the spin-spin relaxation time T2 is influenced by the distance between the nuclei and their mobility. Hence, the magnetization decay depends on the temperature, the crystal polymorph and the crystal size.

\section{Indirect Method}

The indirect method only considers the signal of the liquid fat $70 \mu \mathrm{s}$ after the $90^{\circ}$ pulse and therefore avoids the impact of the crystalline state. However, a second measurement of the melted sample is required for calculating the SFC. After melting for $30 \mathrm{~min}$ at $60{ }^{\circ} \mathrm{C}$ we exposed the sample for $3 \mathrm{~min}$ to crystallization temperature prior to remeasuring. The time was short enough for temperature adaption without recrystallization, making temperature correction unnecessary. The indirect method is accurate but more time consuming and is generally used as a reference method for verifying the quicker direct method.

\section{Approximated SFC in Chocolate}

For chocolate we used the simplified method as described by Ziegleder et al. [33] for measuring the SFC. It is based on the direct method. Due to the additional non-fat solid components a "solid proton content" resulted instead of the SFC. A second measurement of the molten sample was necessary for calculating the approximated SFC as given in Eq. 20. Some systematic error might come about as the ingredients influence the result as a whole.

$\mathrm{SFC}_{\mathrm{a}}=\frac{\left(N_{T}-N_{M}\right) \times 100}{100-N_{M}}$

$\mathrm{SFC}_{\mathrm{a}}$ is the weight percent of crystalline fat at measuring temperature $T$ based on the fat content of the chocolate. $N_{T}$ and $N_{M}$ represent the solid proton content of the crystallizing and the molten sample at measuring temperature.

\section{Results and Discussion}

Polypropylene Crystallization Data Published in the Literature

There is extensive experimental data published in literature on isothermal crystallization kinetics where only Avarami's approximate analytical equation is widely used instead of his theoretical equations. Consequently, the present work analyzes the published experimental data on isothermal crystallization kinetics involving three-dimensional growth of spherulite shaped crystals and uses the corresponding Avrami's theoretical equation. Figure 1 shows the experimental (symbols) increase with time in the relative fraction of crystallinity during the isothermal crystallization of polypropylene under quiescent conditions at different temperatures measured by Manchado et al. [24]. These authors investigated spherulite-shaped, three-dimensional crystal growth using optical polarizing microscopy and found a linear increase in the radius of the spherulite with time, the rate of growth $i$ is listed in Table 1 . The figure also shows the variations obtained by fitting the data to Avrami's theoretical Eq. 16 (continuous lines) for threedimensional growth of crystals in addition to the approximate Eq. 19 (dashed lines). Although they overlap, it is clear that only Avrami's theoretical equation for the threedimensional growth describes well the experimental data of the spherulite shaped polypropylene crystals during isothermal crystallization. This is confirmed by the fact that the regression coefficient is higher and the sum of squares of the errors is smaller than for the one- or two-dimensional growth. Avrami's approximate equation also fits the data well although its quality of fit is not as good as the theoretical equation. Additionally, Avrami's theoretical equation also enables us to determine the physical

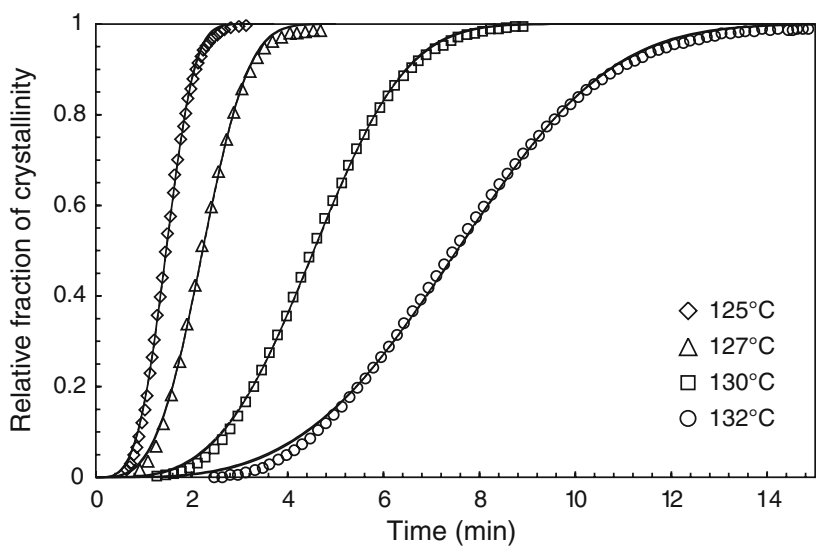

Fig. 1 Isothermal crystallization of polypropylene under static conditions at different temperatures (symbols: data of Manchado et al. [24]). Comparison of Avrami's 3-D theoretical (continuous line) and approximate equation (dashed line) 
Table 1 Values of fitted parameters for the sphere-like (3-D) crystal growth model of Avrami for isothermal polypropylene crystallization at different temperatures corresponding to Fig. 1 (data of Manchado et al. [24])

\begin{tabular}{|c|c|c|c|c|}
\hline \multirow[t]{2}{*}{ Parameter } & \multicolumn{4}{|c|}{ Temperature $\left({ }^{\circ} \mathrm{C}\right)$} \\
\hline & 125 & 127 & 130 & 132 \\
\hline \multicolumn{5}{|c|}{ Experimental value } \\
\hline$i\left(\mu \mathrm{m} \min ^{-1}\right)$ & 16.2 & 10.9 & 5.60 & 3.60 \\
\hline \multicolumn{5}{|c|}{ 3-D crystal growth } \\
\hline$p\left(\min ^{-1}\right)$ & 2.028 & 2.715 & 1.183 & 0.6853 \\
\hline $6 \sigma_{3} \dot{l}^{3} N_{0} / p^{3}$ & 0.350 & 0.0294 & 0.0439 & 0.0505 \\
\hline$N_{0}\left(\mathrm{ml}^{-1}\right)$ & $27.3 \times 10^{6}$ & $18.0 \times 10^{6}$ & $16.4 \times 10^{6}$ & $13.9 \times 10^{6}$ \\
\hline $\begin{array}{l}\text { Regression } \\
\text { coefficient }\end{array}$ & 0.9987 & 0.9988 & 0.9995 & 0.9990 \\
\hline \multicolumn{5}{|c|}{ Approximate equation } \\
\hline$m$ & 3.60 & 3.39 & 3.42 & 3.44 \\
\hline$k\left(\min ^{-m}\right)$ & 0.174 & 0.0459 & 0.00388 & 0.000669 \\
\hline $\begin{array}{l}\text { Regression } \\
\text { coefficient }\end{array}$ & 0.9983 & 0.9984 & 0.9992 & 0.9986 \\
\hline
\end{tabular}

parameters of crystallization such as the probability for growth of nuclei $p$ and the number density of nuclei $N_{0}$ formed initially as listed in Table 1 . As expected, the probability of nucleation $p$, the rate of growth of crystal radius $i$ and the number density of nuclei $N_{0}$ decrease with increase in temperature. In contrast, the determination of these parameters using Avrami's approximate equation cannot be accurate since the index $m$ is usually not an integer value. However, from the fitted values between 3 and 4, a 3-D growth can be expected.

\section{Present Results}

The kinetics of the crystallization of seeded cocoa butter was investigated in the present work for the first time. In both unseeded and seeded cocoa butter samples, the solidification progressed in two steps. At higher temperature, these steps are more strongly pronounced. Compared to the final SFC, the quantity of the initially crystallizing fraction was found to be up to $20 \%$ (Figs. 3, 4, 5 and 6).

These findings are in accordance with the work of Dimick and Manning [34] who investigated quiescent crystallization of not precrystallized cocoa butter between 26 and $33{ }^{\circ} \mathrm{C}$. In the same sample they observed the formation of crystals of different shape and TAG composition.

We were able to show in experiments using Differential Scanning Calorimetry and X-Ray Diffraction (XRD) analysis (unpublished data) that a fraction of higher melting triglycerides can form. It is most probable that the fractionation corresponds to higher melting SOS triglycerides. XRD revealed a peak corresponding to a short spacing of $3.86 \AA$, which is close to the peak of strongest intensity measured by
Sato et al. [35] for SOS in form $\gamma$. Like form $\beta_{\mathrm{V}}$ of cocoa butter, $\gamma$-SOS is stacked in a triple chain length. However, as the sub-cell is orthorhombic, the triclinic crystallization of $\beta_{\mathrm{V}}$ in the bulk is neither induced nor accelerated by this fraction. Similar observations are reported by other authors $[5,34]$ for statically crystallized cocoa butter in the melting range of the $\beta_{\mathrm{V}}$ crystal form $\left(25-33{ }^{\circ} \mathrm{C}\right)$. Therefore we treated the two main steps as independent crystallization events. We applied a double Avrami-fit [14] to the data where the bimodal crystallization was developed. No discrimination was performed if the primary fractionation crystallization was not clearly distinguishable (Fig. 5).

The viscosity relates to the molecular interaction and the diffusion, which control the crystallization of a melt. Without any crystalline fractions, a Newtonian fluid behavior and a distinct temperature dependency are observed in fat melts. The exponential relation between temperature $T$ and viscosity $\eta$ is given by

$\eta=A \cdot \mathrm{e}^{E_{a} / R T}$

where the factor $A$ is a measure for the molecular interaction and $E_{a}$ is the activation energy. For visualization an Arrhenius plot is appropriate. Figure 2 shows the viscosity of cocoa butter between 20 and $50{ }^{\circ} \mathrm{C}$. Above $27.5^{\circ} \mathrm{C}$ we observed that the data fitted well to Eq. 21. The activation energy $E_{a}$ represented by the slope of the dashed line is $23 \mathrm{~kJ} \mathrm{~mol}^{-1}$, which is close to the value reported in the literature [36].

The deviation from the linear curve progression below $27.5^{\circ} \mathrm{C}$ can be attributed to a decreasing mobility of the TAG molecules, as by preorientation or even the onset of nucleation.

We used a temperature controlled plate-plate geometry with a diameter of $25 \mathrm{~mm}$ at a shear rate of $150 \mathrm{~s}^{-1}$ to allow for temperature adaption within a few seconds. The impact of shear might also significantly shorten the induction time of nucleation.

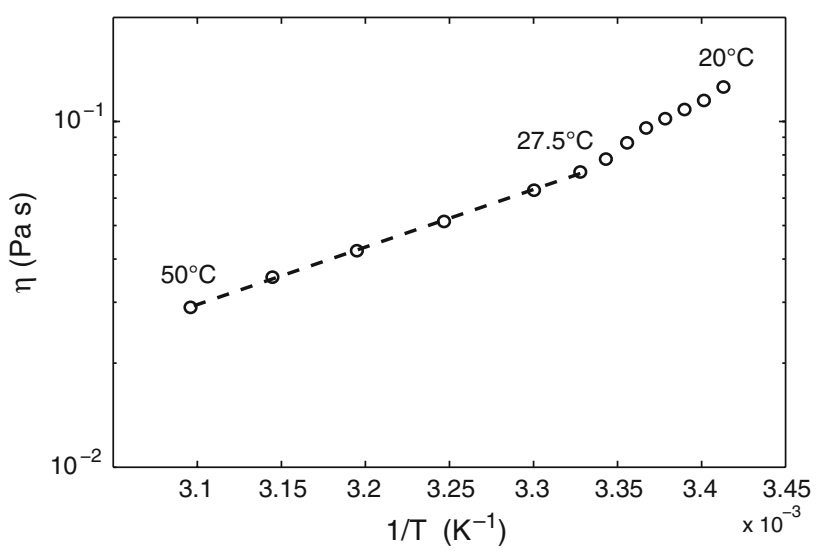

Fig. 2 Temperature dependency of the cocoa butter viscosity. Arrhenius fit between 50 and $27.5^{\circ} \mathrm{C}$ (dashed line) 


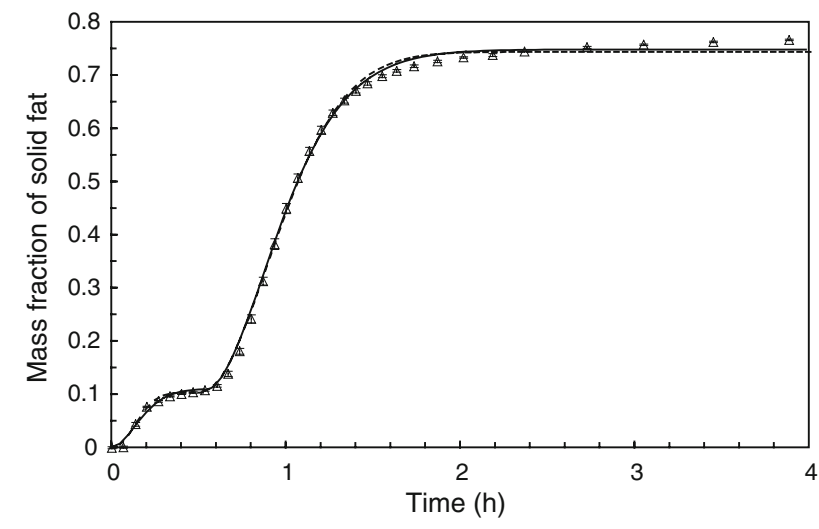

Fig. 3 Isothermal crystallization of unseeded cocoa butter at $20{ }^{\circ} \mathrm{C}$ (triangles with standard deviation) under static conditions. Comparison of Avrami's 1-D theoretical (continuous line) and approximate equation (dashed line) when applying double-Avrami fits

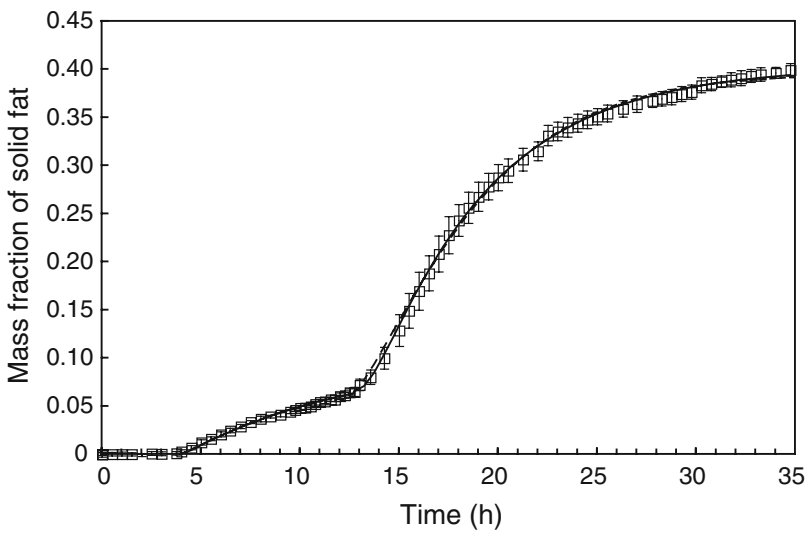

Fig. 4 Isothermal crystallization of unseeded cocoa butter at $25{ }^{\circ} \mathrm{C}$ (squares with standard deviation) under static conditions. Comparison of Avrami's 1-D theoretical (continuous line) and approximate equation (dashed line) when applying double-Avrami fits

\section{Unseeded Cocoa Butter}

The experimental data on the time dependent evolution of the SFC for unseeded cocoa butter at 20 and $25{ }^{\circ} \mathrm{C}$ are given in Figs. 3 and 4, respectively. There is clear bimodal crystallization behavior, which allows us to apply doubleAvrami fits. The parameters obtained by fitting the main crystallization event to the 1-D theoretical model of Avrami and the approximate equation are listed in Table 2. Since the 2-D and 3-D models poorly fitted the data, a lineal crystal growth is evident. The major crystallization domain will consist of crystals in form $\alpha$ at $20{ }^{\circ} \mathrm{C}$ and form $\beta_{\mathrm{IV}}$ at $25^{\circ} \mathrm{C}$. However, the beginning of $\alpha$ to $\beta_{\mathrm{IV}}$ transformation at $20{ }^{\circ} \mathrm{C}$ is possible.

At $20^{\circ} \mathrm{C}$ the two crystallization steps can easily be separated. At $25{ }^{\circ} \mathrm{C}$ however, there is a gradual transition from the fraction crystallization to the main crystallization
Table 2 Values of fitted parameters for the rod-like (1-D) crystal growth model of Avrami for isothermal crystallization of unseeded cocoa butter bulk phase at 20 and $25{ }^{\circ} \mathrm{C}$ corresponding to Figs. 3 and 4

\begin{tabular}{llc}
\hline Parameter & \multicolumn{2}{l}{ Temperature $\left({ }^{\circ} \mathrm{C}\right)$} \\
\cline { 2 - 3 } & 20 & 25 \\
\hline Initial conditions & & \\
$S_{m 0}$ & 0.106 & 0.0647 \\
$t_{0}(\mathrm{~h})$ & 0.533 & 12.8 \\
Rod-like 1-D crystal growth & & \\
$S_{m}-S_{m 0}$ & 0.642 & 0.338 \\
$p\left(\mathrm{~h}^{-1}\right)$ & 1.59 & 0.938 \\
$\sigma_{1} i N_{0} / p$ & 3.40 & 0.183 \\
Regression coefficient & 0.9981 & 0.9988 \\
Approximate equation & & \\
$S_{m}-S_{m 0}$ & 0.637 & 0.330 \\
$m$ & 1.83 & 1.26 \\
$k\left(\mathrm{~h}^{-m}\right)$ & 2.99 & 0.0901 \\
Regression coefficient & 0.9973 & 0.9974 \\
\hline
\end{tabular}

of the bulk. We conclude that the two crystallization events have different induction times. Before nucleation starts, a stationary distribution of embryos has to be established which is observed by the presence of an induction time. As described by Rousset [6], the induction time for large molecules can be considerable as the reorientation of the molecules depends on their mobility. At higher temperature however, the impact of Brownian motion also promotes the stochastic arrangement, resulting in a considerable increase of the induction time. As can be seen in Table 2, the probability of nucleation $p$ of $\alpha$ crystals at $20{ }^{\circ} \mathrm{C}$ is higher than that of $\beta_{\mathrm{IV}}$ crystals at $25{ }^{\circ} \mathrm{C}$.

\section{Seeded Cocoa Butter}

The cocoa butter seed crystals from the single-stage device develop a "sheaf" shaped or flake like morphology during conditioning. Under conventional processing conditions the size of the crystals from the single-stage system is larger (number based median of $7.5 \mu \mathrm{m}$ ) than from the continuous two-stage crystallizer $(4.1 \mu \mathrm{m})$ as shown by Mehrle [37].

The results of quiescent crystallization of seeded cocoa butter at 28,30 and $32{ }^{\circ} \mathrm{C}$ are given in Figs. 5 and 6 . We used the direct method for measuring the SFC in this series. At higher temperatures, the two-step solidification behavior is more strongly pronounced. We applied double-Avrami fits to the experimental data at $32{ }^{\circ} \mathrm{C}$ for both the approximate and 1-D theoretical equations. This was not possible for 28 and $30{ }^{\circ} \mathrm{C}$ since the start of bulk crystallization overlapped the fraction crystallization. For 


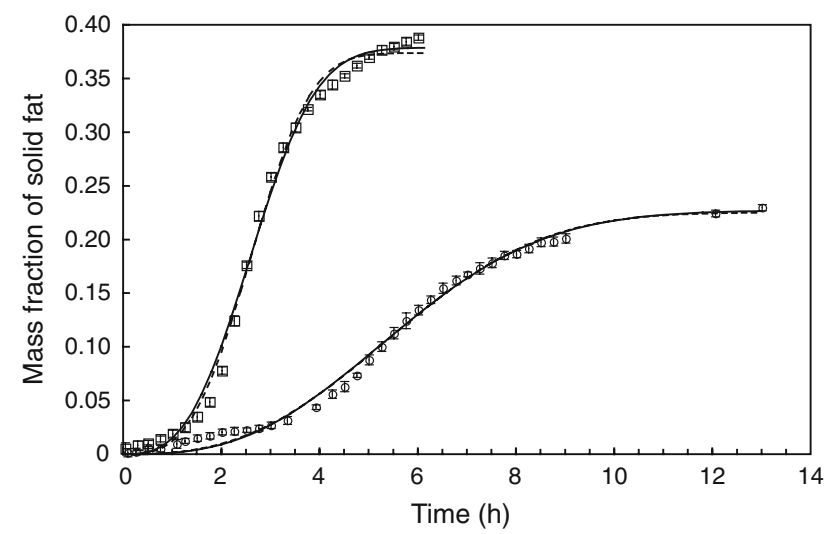

Fig. 5 Isothermal crystallization of seeded cocoa butter at $28{ }^{\circ} \mathrm{C}$ (squares with standard deviation) and $30{ }^{\circ} \mathrm{C}$ (circles with standard deviation) under static conditions. Comparison of Avrami's 2-D theoretical (continuous line) and approximate equation (dashed line)

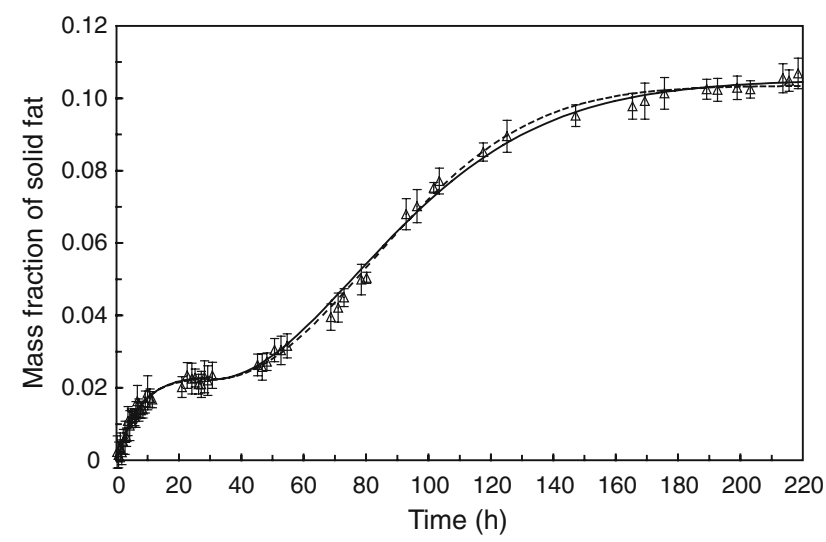

Fig. 6 Isothermal crystallization of seeded cocoa butter at $32{ }^{\circ} \mathrm{C}$ (triangles with standard deviation) under static conditions. Comparison of Avrami's 1-D theoretical (continuous line) and approximate equation (dashed line) when applying double-Avrami fits

these experiments, the solidification showed the best agreement with the 2-D growth of Avrami's theoretical equation. However, even for the 2-D model, the 95\% intervals of the fitted parameters are substantial, so that we cannot declare the Avrami model to be appropriate for quantifying the crystallization of seeded cocoa butter samples.

The experimental data shown in Figs. 5 and 6 are represented in a different way in Fig. 7. By double logarithmic transformation of Eq. 19, Avrami's approximate equation can be linearized as follows

$\ln \left(-\ln \left(1-\frac{\mathrm{SFC}}{\mathrm{SFC}_{m}}\right)\right)=\ln (k)+m \ln (t)$

This allows us to visualize the time dependency of the crystallization kinetics (Avrami exponent $\mathrm{m}$ ) by variation of the slope. It can be seen that a similar slope exists for all three temperatures when the fraction crystallizes

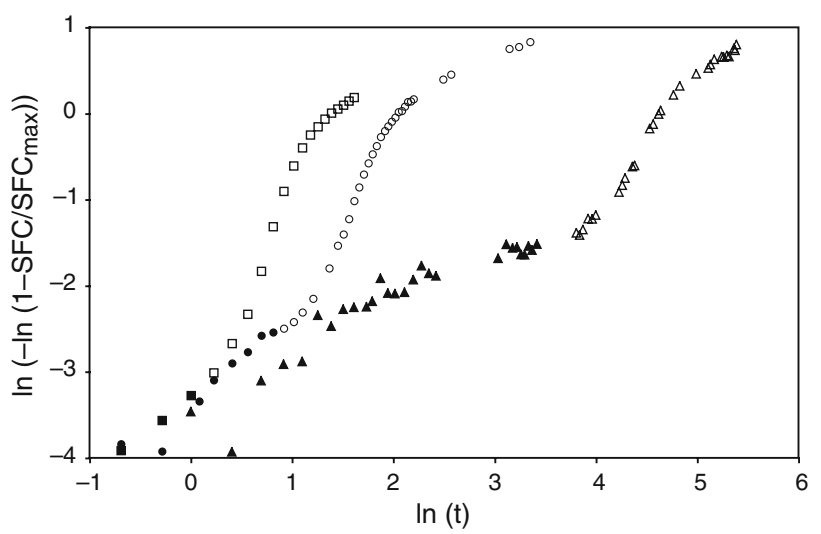

Fig. 7 Isothermal crystallization of seeded cocoa butter at $28{ }^{\circ} \mathrm{C}$ (squares), $30{ }^{\circ} \mathrm{C}$ (circles) and $32{ }^{\circ} \mathrm{C}$ (triangles) in the rearranged double logarithmic representation. Differentiation in the initial fraction crystallization (closed symbols) and the subsequent bulk crystallization (open symbols)

(closed symbols) at the beginning. A 1-D growth kinetics seems to fit quite well, even though scattering is more strongly pronounced at low solids content. The subsequent bulk crystallization (open symbols) starts with a higher order growth which is continuously decreasing. This was also observed during isothermal unseeded palm oil crystallization by $\mathrm{Ng}$ and $\mathrm{Oh}$ [38]. Hence, a transition from 3-D to 1-D crystal growth could be assumed. The same conclusion can be drawn from the data in Fig. 5. At the maximum growth rate the slope in the experiment is steeper than the 2-D Avrami fit indicating a higher order growth. When approaching the maximum volume fraction of crystalline phase the experimental data show a lower flattening tendency compared to the fitting models, indicating a transition to a lower rate of growth. The transition takes place during bulk crystallization after $3 \mathrm{~h}$ (26\% SFC) at $28{ }^{\circ} \mathrm{C}, 7.5 \mathrm{~h}(17.5 \% \mathrm{SFC})$ at $30{ }^{\circ} \mathrm{C}$ and $130 \mathrm{~h}(9.2 \% \mathrm{SFC})$ at $32{ }^{\circ} \mathrm{C}$. In long term observations the SFC rose above $50 \%$ at $28{ }^{\circ} \mathrm{C}$ and $29 \%$ at $30{ }^{\circ} \mathrm{C}$ after 3 days. We conclude that, the growth rate already changes after half of the bulk phase crystallizes. Impediment of the growing crystals, purification and removal of imperfections in the crystal lattice driven by diffusion and the onset of $\beta_{\mathrm{V}}$ to $\beta_{\mathrm{VI}}$ transformation are possible effects of this phenomenon.

Additional microstructural information using optical microscopy is necessary to determine the dimensionality of growth. The crystal growth arising from a seed crystal at $23{ }^{\circ} \mathrm{C}$ observed using optical microscopy is displayed in Fig. 8. Although a three-dimensional (spherulitic) growth of fat crystals occurs, the sub-units of the fat crystals have a hair-like morphology. Depending on the crystallization rate, the crystalline filaments can be more densely packed or build a looser assembly. A high 
Fig. $8 \beta_{\mathrm{V}}$ crystallization at $23{ }^{\circ} \mathrm{C}$ on a cocoa butter seed crystal (single-stage). Time: left $0 \mathrm{~min}$, center $8 \mathrm{~min}$, right $13 \mathrm{~min}$

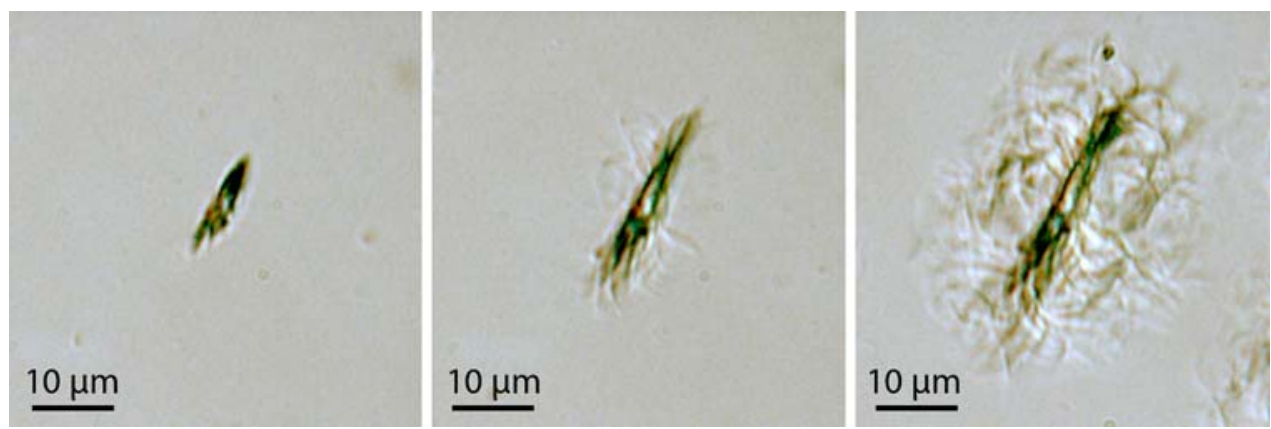

branching probability would result in an increased order crystallization kinetics.

A high portion of liquid fat appears to be enclosed in the spherical shaped crystal entity. Consequently, the 3-D approximation of the crystallization by the Avrami model is not realistic. In fact, no theoretical model exists for such complex multi component TAG systems. The development of any other type of model would depend on more fundamental mechanistic information such as diffusion of the TAGs, orientation, the cooling rate to reach the crystallization temperature as well as material specific knowledge about the TAG composition and other components (free fatty acids, mono- and diacylglycerides). Additionally, precrystallization (number and size distribution of the seed crystals) will influence the growth kinetics. Although the seed concentration is the same, the induction time before bulk crystallization starts is higher. The preorientation time of the TAG molecules is longer at higher temperatures. This agrees with the formation of a lamellar arrangement of the TAGs in the melt before crystallization can start, as proposed by Larsson [39].

\section{Milk Chocolate}

Different methods of precrystallization are compared with respect to isothermal crystallization of chocolate at $28{ }^{\circ} \mathrm{C}$. For all measurements Avrami's 1-D theoretical equation fits well with the experimental data. In Fig. 9 the results of the indirect and the direct measured variations of the SFC of conventional precrystallized chocolate are compared. Averaging of the solidification curves of indirect measured samples was omitted due to the delay between the single measurements. Similar parameters for the maximum mass fraction of crystalline phase $S_{m}$, probability of nucleation $p$ and the rate constant $\sigma_{1} i N_{0}$ were evaluated for both methods as given in Table 3. Since we found no significant difference between the two NMR measuring methods, the approximated SFC was computed in the subsequent experiments using Eq. 20 based on the direct method.

Figure 10 compares the results of the seeded milk chocolate using the single-stage batch process (triangles)

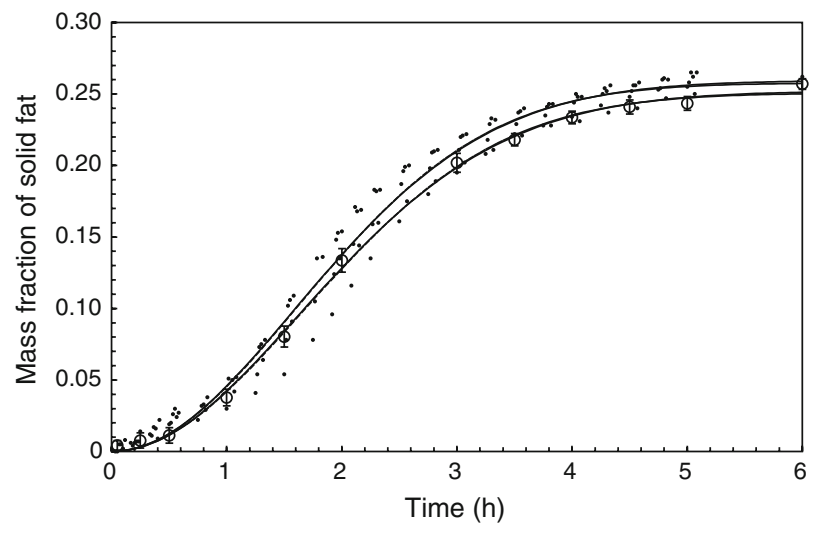

Fig. 9 Isothermal fat crystallization in conventional tempered milk chocolate at $28{ }^{\circ} \mathrm{C}$ under static conditions. Comparison between the direct (circles with standard deviation) and the indirect measuring method (dots) for SFC quantification. Avrami's 1-D theoretical (continuous line) and approximate equation (dashed line)

and the continuous two-stage process (squares). Due to the overlapping standard deviation within the measurements, we found no significant difference between the two techniques of seed crystal formation. As expected, the mass fraction of seed crystals can be reduced without significantly changing the solidification kinetics when a smaller grain size is added. The values of $\sigma_{1} i N_{0}$ obtained from Table 3 are about the same for conventional precrystallized chocolate and for single-stage seeded samples. However, the numerical values of the fitted parameters should be interpreted with prudence as there is a certain standard deviation within the measurements.

The favored 1-D growth of the crystals might be caused by the high concentration of solid ingredients present in chocolate. The molten milk chocolate we used has a volume fraction of $56 \%$ solids. Hence, spherulitic 3-D crystal growth is impeded, especially by the sugar crystals, cocoa fibers and milk proteins. Additionally, if we assume regions to be present in which the continuous fat phase is immobilized (e. g. in milk powder particles), the free volume for crystal growth is further reduced. As a consequence, 1-D growth of the crystals is a reasonable finding. 
Table 3 Values of fitted parameters for the rod-like (1-D) crystal growth model of Avrami for transient SFC measurement during isothermal crystallization of precrystallized milk chocolate at $28{ }^{\circ} \mathrm{C}$

Conventional tempering: comparison of SFC measuring methods (Fig. 9),

Precrystallization by seeding: comparison of different seeding devices (Fig. 10)

\begin{tabular}{|c|c|c|c|c|}
\hline \multirow{2}{*}{$\begin{array}{l}\text { Precrystallization method } \\
\text { Comparison of }\end{array}$} & \multicolumn{2}{|c|}{ Conventional tempering } & \multicolumn{2}{|l|}{ Seeding } \\
\hline & Indirect SFC & Direct SFC & Two-stage & Single-stage \\
\hline \multicolumn{5}{|l|}{ Rod-like 1-D crystal growth } \\
\hline$S_{m}$ & 0.259 & 0.252 & 0.2466 & 0.259 \\
\hline$p\left(\mathrm{~h}^{-1}\right)$ & 0.0817 & 0.103 & 0.313 & 0.148 \\
\hline$\sigma_{1} i N_{0} / p$ & 59.8 & 35.9 & 5.07 & 20.9 \\
\hline Regression coefficient & 0.9853 & 0.9985 & 0.9983 & 0.9963 \\
\hline \multicolumn{5}{|l|}{ Approximate equation } \\
\hline$S_{m}$ & 0.258 & 0.251 & 0.244 & 0.256 \\
\hline$m$ & 1.98 & 1.96 & 1.86 & 2.00 \\
\hline$k\left(\mathrm{~h}^{-m}\right)$ & 0.194 & 0.183 & 0.227 & 0.212 \\
\hline Regression coefficient & 0.9853 & 0.9984 & 0.9980 & 0.9964 \\
\hline
\end{tabular}

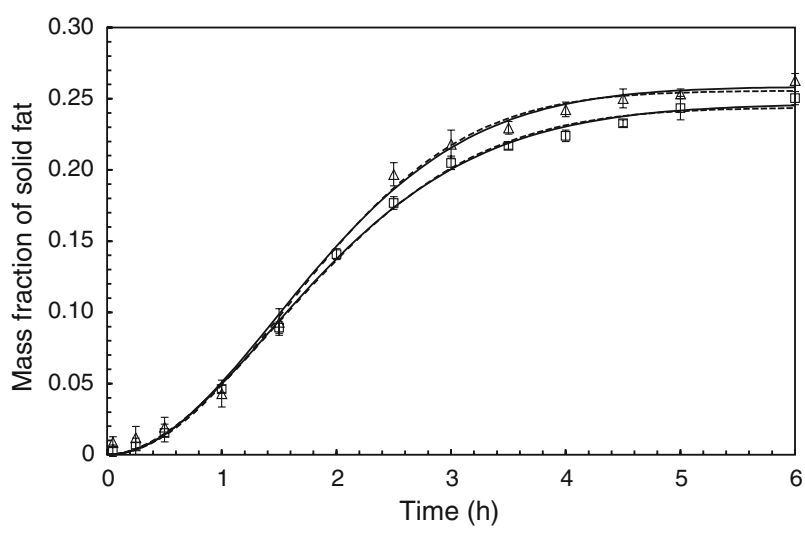

Fig. 10 Isothermal fat crystallization in seeded milk chocolate at $28{ }^{\circ} \mathrm{C}$ under static conditions. Comparison between the single-stage (triangles with standard deviation) and the two-stage device (squares with standard deviation). Avrami's 1-D theoretical (continuous line) and approximate equation (dashed line)

\section{Conclusions}

From a descriptive point of few, it is of interest to quantify crystallization processes with physical parameters as given in Avrami's theoretical equations for one-, two- and three-dimensional crystal growth. Although Avrami's approximate equation usually fits the sigmoidal crystallization well in the experiments, the two parameters involved do not represent physical properties. The validity of Avrami's theoretical equation for 3-D growth was verified with data from the literature on polypropylene crystallization.

Solidification of unseeded and seeded cocoa butter revealed the complexity of this fat system. We observed a time lag before crystallization started and the segregation of a crystalline fraction. At a low solids growth rate of cocoa butter crystals in form $\beta_{\mathrm{V}}$ (no precrystallization, high temperature) the fractionation is more strongly pronounced. The kinetics of crystallization for unseeded cocoa butter in form $\alpha$ at $20{ }^{\circ} \mathrm{C}$ and $\beta_{\mathrm{IV}}$ at $25{ }^{\circ} \mathrm{C}$ are well represented by Avrami's 1-D theoretical equation.

For pure cocoa butter crystallization of seeded samples, we observed a transition from 3-D to 1-D growth during the main crystallization event. This might be a result of the increasing sterical hindrance of the growing crystals when approaching the final SFC. Solidification of precrystallized chocolate at $28{ }^{\circ} \mathrm{C}$ is found to be best described by a 1-D growth. The dispersed solid particles significantly determine the crystallization kinetics of the continuous fat phase. Moreover, we have shown that there is no significant difference between different SFC measuring methods and precrystallization processes for a commercial milk chocolate. In general, Avrami's model did not describe the crystallization kinetics of precrystallized cocoa butter and chocolate.

Cocoa butter has a narrower triglyceride spectrum compared to other vegetal or animal fats. However, especially at higher temperatures, a complex crystallization behavior is pronounced. Obviously, the overall phenomenology observed during the phase change at different temperatures cannot be reduced to a single equation. Especially for higher crystallization temperatures, the side effects such as fractionation, refinement of the crystal lattice and crystal transformation gain in impact.

Acknowledgments We are grateful to Prof. B. Patterson from the Paul Scherrer Institute (Villigen, Switzerland) and Dr. P. Panine from ESRF (Grenoble, France) for providing the X-ray facility for crystal form determination. The authors also thank Dr. P. Braun (Bühler AG, Switzerland) for providing the seeding equipment and H. Mikle (Chocolats Halba AG, Switzerland) for analyzing the TAG composition of the cocoa butter.

\section{References}

1. Mazzanti G, Guthrie SE, Sirota EB, Marangoni AG, Idziak SHJ (2003) Orientation and phase transitions of fat crystals under shear. Cryst Growth Des 3(5):721-725 
2. Wille RL, Lutton ES (1966) Polymorphism of cocoa butter. J Am Oil Chem Soc 43(8):491-496

3. Lovegren NV, Gray MS, Feuge RO (1976) Effect of liquid fat on melting-point and polymorphic behavior of cocoa butter and a cocoa butter fraction. J Am Oil Chem Soc 53(3):108-112

4. Himawan C, Starov VM, Stapley AGF (2006) Thermodynamic and kinetic aspects of fat crystallization. Adv Colloid Interface Sci 122(1-3):3-33

5. Marangoni AG, McGauley SE (2003) Relationship between crystallization behavior and structure in cocoa butter. Cryst Growth Des 3(1):95-108

6. Rousset P (2002) Modelling crystallization kinetics of triacylglycerols. In: Physical properties of lipids. Marcel Dekker, Inc, pp 1-36

7. Foubert I, Vanrolleghem PA, Vanhoutte B, Dewettinck K (2002) Dynamic mathematical model of the crystallization kinetics of fats. Food Res Int 35(10):945-956

8. Foubert I, Dewettinck K, Vanrolleghem PA (2003) Modelling of the crystallization kinetics of fats. Trends Food Sci Technol 14(3):79-92

9. Avrami M (1939) Kinetics of phase change. I general theory. J Chem Phys 7(12):1103-1112

10. Avrami M (1940) Kinetics of phase change. II transformationtime relations for random distribution of nuclei. J Chem Phys 8(2):212-224

11. Mandelkern L, Quinn FA Jr, Flory PJ (1954) Crystallization kinetics in high polymers. I. bulk polymers. J Appl Phys 25(7):830-839

12. Kawamura K (1979) The DSC thermal analysis of crystallization behavior in palm oil. J Am Oil Chem Soc 56(8):753-758

13. Sharples A (1966) Introduction to polymer crystallization. Edward Arnold, Ltd., London

14. Marangoni AG (2005) Crystallization kinetics. In: Fat crystal networks. Marcel Dekker, Inc., New York, pp 21-82

15. Christian JW (2002) The theory of transformations in metals and alloys. Number Part I \& Part II, 3rd edn. Elsevier Science Ltd., Pergamon

16. Pradell T, Crespo D, Clavaguera N, Clavaguera-Mora MT (1998) Diffusion controlled grain growth in primary crystallization: Avrami exponents revisited. J Phys Condens Matter 10(17):3833-3844

17. Ng WL (1975) Thermal decomposition in the solid state. Aust J Chem 28(6):1169-1178

18. Khanna YP, Taylor TJ (1988) Comments and recommendations on the use of the Avrami-equation for physicochemical kinetics. Polym Eng Sci 28(16):1042-1045

19. Marangoni AG (1998) On the use and misuse of the Avrami equation in characterization of the kinetics of fat crystallization. J Am Oil Chem Soc 75(10):1465-1467

20. Liu XY, Sawant PD (2002) Mechanism of the formation of selforganized microstructures in soft functional materials. Adv Mater 14(6):421-426

21. Marangoni AG, Ollivon M (2007) Fractal character of triglyceride spherulites is a consequence of nucleation kinetics. Chem Phys Lett 442(4-6):360-364
22. Berg TGO, Brimberg UI (1983) Kinetics of fat crystallization. Fette Seifen Anstrichmittel 85(4):142-149

23. Kloek W, Walstra P, van Vliet T (2000) Nucleation kinetics of emulsified triglyceride mixtures. J Am Oil Chem Soc 77(6):643652

24. Manchado MAL, Biagiotti J, Torre L, Kenny JM (2000) Effects of reinforcing fibers on the crystallization of polypropylene. Polym Eng Sci 40(10):2194-2204

25. Long Y, Shanks RA, Stachurski ZH (1995) Kinetics of polymer crystallization. Prog Polym Sci 20(4):651-701. ISSN 0079-6700

26. Supaphol P, Spruiell JE (2000) Application of the Avrami, Tobin, Malkin, and simultaneous Avrami macrokinetic models to isothermal crystallization of syndiotactic polypropylenes. J Macromol Sci Phys B 39(2):257-277

27. Zeng Y (2000) Impf- und Scherkristallisation von Schokoladen. Diss. ETH Nr. 13798, ETH Zürich

28. Beckett ST (2003) Industrial chocolate manufacture and use, 3 edn. Blackwell, Oxford

29. AOCS Official Method Cd 16b-93 (1999) Solid fat content (SFC) by low-resolution nuclear magnetic resonance-the direct method

30. AOCS Official Method Cd 16b-93 (1999) Solid fat content (SFC) by low-resolution nuclear magnetic resonance-the indirect method

31. van Putte K, van den Enden JC (1974) Fully automated determination of solid fat content by pulsed $\mathrm{NMR}^{1}$. J Am Oil Chem Soc 51:316-320

32. Timms RE (2003) Confectionery fats handbook (properties, production and application). The oily press lipid library, vol 14, 1 edn. P.J. Barnes \& Associates, Bridgewater

33. Ziegleder G, Moser C, Geier-Greguska J (1996) Kinetics of fat migration within chocolate products, part II: influence of storage temperature, diffusion coefficient, solid fat content. Fett-Lipid 98(7-8):253-256

34. Dimick PS, Manning DM (1987) Thermal and compositional properties of cocoa butter during static crystallization. J Am Oil Chem Soc 64(12):1663-1669

35. Sato K, Arishima T, Wang ZH, Ojima K, Sagi N, Mori H (1989) Polymorphism of POP and SOS. 1. Occurrence and polymorphic transformation. J Am Oil Chem Soc 66(5):664-674

36. Weipert D, Tscheuschner H-D, Windhab E (1993) Rheologie der Lebensmittel. Behr's Verlag GmbH, Hamburg

37. Mehrle YE (2007) Solidification and contraction of confectionery systems in rapid cooling processing. Diss. ETH Nr. 17443, ETH Zurich

38. Ng WL, Oh CH (1994) A Kinetic Study on isothermal crystallization of palm oil by solid fat content measurements. J Am Oil Chem Soc 71(10):1135-1139

39. Larsson K (1972) Molecular arrangements in glycerides. Fette Seifen Anstrichmittel 74(3):136-142 